\title{
Editorial review board
}

Ernie Cadotte, University of Tennessee

Elissa Grossman, University of Southern California

Jerry Katz, St. Louis University

Heidi Neck, Babson College

Susana Santos, Rowan University

Mark Schenkel, Belmont University

Julie Shields, Millikin University

George Solomon, George Washington University

Mark Weaver, University of South Alabama

Rebecca White, University of Tampa

Christoph Winkler, Iona College 